\title{
Existence of Solutions for the Debye-Hückel System with Low Regularity Initial Data
}

\author{
Jihong Zhao ${ }^{1}$, Qiao $\mathrm{Liu}^{2}$, and Shangbin Cui ${ }^{2 *}$ \\ ${ }^{1}$ Department of Mathematics, Northwest A\&F University, Yangling, \\ Shaanxi 712100, People's Republic of China \\ 2 Department of Mathematics, Sun Yat-sen University, Guangzhou, \\ Guangdong 510275, People's Republic of China
}

\begin{abstract}
In this paper we study existence of solutions for the Cauchy problem of the Debye-Hückel system with low regularity initial data. By using the CheminLerner time-space estimate for the heat equation, we prove that there exists a unique local solution if the initial data belongs to the Besov space $\dot{B}_{p, q}^{s}\left(\mathbb{R}^{n}\right)$ for $-\frac{3}{2}<s \leq-2+\frac{n}{2}, p=\frac{n}{s+2}$ and $1 \leq q \leq \infty$, and furthermore, if the initial data is sufficiently small then the solution is global. This result improves the regularity index of the initial data space in previous results on this model.
\end{abstract}

Keywords: Debye-Hückel system; low regularity; global existence.

Mathematics Subject Classification 2010: $35 \mathrm{~K} 45,35 \mathrm{Q} 99$

\section{Introduction}

In this paper we study the following Debye-Hückel system arising from the theory of electrolytes ([6]):

$$
\begin{cases}\partial_{t} v=\nabla \cdot(\nabla v-v \nabla \phi) & \text { in } \mathbb{R}^{n} \times(0, \infty), \\ \partial_{t} w=\nabla \cdot(\nabla w+w \nabla \phi) & \text { in } \mathbb{R}^{n} \times(0, \infty), \\ \Delta \phi=v-w & \text { in } \mathbb{R}^{n} \times(0, \infty), \\ v(x, 0)=v_{0}(x), \quad w(x, 0)=w_{0}(x) & \text { in } \mathbb{R}^{n},\end{cases}
$$

where $v$ and $w$ denote the densities of the electron and the hole, respectively, in an electrolytes, and $\phi$ denotes the electric potential.

Mathematical analysis of the Debye-Hückel system was first focused on the initial boundary value problems in 1980's, and some results related to the global existence,

*E-mail: zhaojihong2007@yahoo.com.cn; liuqao2005@163.com; cuisb3@yahoo.com.cn. 
uniqueness and regularity of classical solutions and the asymptotic stability of stationary solutions were obtained by using the Green function, the Poincaré inequality and the standard maximum principle of equations of parabolic type; see [7], 8], [12] and [15] for more details. In 1994, Biler, Hebisch and Nadzieja in [3] considered the no-flux boundary problem of (1.1), and considered global and local existence of weak solutions, convergence rate estimates to stationary solutions of time-dependent solutions, and for further studies related to this topic we refer the reader to see [1], [2] and the references therein.

In 1999, Karch in 9] proved existence and uniqueness of solutions of the problem (1.1) for initial data in the Besov space $\dot{B}_{p, \infty}^{s}\left(\mathbb{R}^{n}\right)$ with $-1<s<0$ and $p=\frac{n}{s+2}$. Note that similar results for initial data in the Lebesgue and Sobolev spaces was established only recently, see the work of Kurokiba and Ogawa [10]. In [13], Ogawa and Shimizu considered existence of global solutions of the problem (1.1) for small initial data in a two-dimensional critical Hardy space. The purpose of this paper is to prove existence of solutions for (1.1) with initial data in the Besov space $\dot{B}_{p, q}^{s}\left(\mathbb{R}^{n}\right)$ of indices $-\frac{3}{2}<s \leq-2+\frac{n}{2}, p=\frac{n}{s+2}$ and $1 \leq q \leq \infty$. This result improves the corresponding result of Karch obtained in [9]. It shows that the Debye-Hückel system has a better property than the Navier-Stokes equations in regard to existence of solutions, since for that equation there is no existence result for initial data in a space with regularity index $s$ smaller than -1 . In fact, the nonlinear term of the system (1.1) seems to be closer to the quadratic nonlinear heat equation $\left(\sim u^{2}\right)$ than to the Navier-Stokes equations $(\sim u \cdot \nabla u)$, and our main result (see Theorem 1.1 below) also holds for the quadratic nonlinear heat equation and is even new for this equation. Main tools used to get our main result are the CheminLerner space $\mathfrak{L}^{r}\left(0, T ; \dot{B}_{p, q}^{-2+n / p+2 / r}\right)$ and some related estimates (see Definition 2.1 and Propositions 2.2 and 2.3 in Section 2).

Note that from the third equation in (1.1) we have

$$
\phi=(-\Delta)^{-1}(w-v):=E *(w-v),
$$

where $E(x)=-\frac{1}{2 \pi} \log |x|$ for $n=2$ and $E(x)=\frac{1}{4} \pi^{-n / 2} \Gamma\left(\frac{n}{2}-1\right)|x|^{-(n-2)}$ for $n \geq 3$, so that we can eliminate $\phi$ from (1.1) and obtain

$$
\begin{cases}\partial_{t} v-\Delta v=-\nabla \cdot\left(v \nabla(-\Delta)^{-1}(w-v)\right) & \text { in } \mathbb{R}^{n} \times(0, \infty), \\ \partial_{t} w-\Delta w=\nabla \cdot\left(w \nabla(-\Delta)^{-1}(w-v)\right) & \text { in } \mathbb{R}^{n} \times(0, \infty), \\ v(x, 0)=v_{0}(x), \quad w(x, 0)=w_{0}(x) & \text { in } \mathbb{R}^{n}\end{cases}
$$

Hence, we only need to consider this equivalent problem. We now give the precise statement of our main result, and for simplicity, we use $(v, w) \in \mathcal{X}$ to denote $(v, w) \in$ $\mathcal{X} \times \mathcal{X}$ for a Banach space $\mathcal{X}$.

Theorem 1.1. Let $n \geq 2,1 \leq q \leq \infty$ and $2 \leq p<2 n$. Suppose that $\left(v_{0}, w_{0}\right) \in$ $\dot{B}_{p, q}^{-2+n / p}\left(\mathbb{R}^{n}\right)$. There exists $T>0$ such that the problem (1.1) has a unique solution

$$
(v, w) \in \underset{1<r \leq \infty}{\cap} \mathfrak{L}^{r}\left(0, T ; \dot{B}_{p, q}^{-2+n / p+2 / r}\left(\mathbb{R}^{n}\right)\right) .
$$


Moreover, if $\left(v_{0}, w_{0}\right)$ belongs to the closure of $\mathcal{S}\left(\mathbb{R}^{n}\right)$ in $\dot{B}_{p, q}^{-2+n / p}\left(\mathbb{R}^{n}\right)$, then $(v, w) \in$ $C\left([0, T), \dot{B}_{p, q}^{-2+n / p}\left(\mathbb{R}^{n}\right)\right)$. Besides, there exists $\varepsilon>0$ such that if $\left\|\left(v_{0}, w_{0}\right)\right\|_{\dot{B}_{p, q}^{-2+n / p}} \leq$ $\varepsilon$, then the above assertion holds for $T=\infty$, i.e., the solution $(v, w)$ is global. Furthermore, if $(v, w)$ and $(\tilde{v}, \tilde{w})$ are two solutions of (1.1) with initial data $\left(v_{0}, w_{0}\right)$ and $\left(\tilde{v}_{0}, \tilde{w}_{0}\right)$, respectively, then there exists a universal constant $C>0$ such that for any $1<r \leq \infty$, we have

$$
\|(v-\tilde{v}, w-\tilde{w})\|_{\mathfrak{L}^{r}\left(0, T ; \dot{B}_{p, q}^{-2+n / p+2 / r}\right)} \leq C\left\|\left(v_{0}-\tilde{v}_{0}, w_{0}-\tilde{w}_{0}\right)\right\|_{\dot{B}_{p, q}^{-2+n / p}}
$$

It is easy to verify that (1.1) is invariant under the scaling $v_{\lambda}(x, t)=\lambda^{2} v\left(\lambda x, \lambda^{2} t\right)$, $w_{\lambda}(x, t)=\lambda^{2} w\left(\lambda x, \lambda^{2} t\right)$ and $\phi_{\lambda}(x, t)=\phi\left(\lambda x, \lambda^{2} t\right)$. Hence, as a standard practice, we have the following existence result for self-similar solution of (1.1):

Corollary 1.2. Let $n \geq 2$ and $\frac{n}{2} \leq p<2 n$. Suppose that $\left(v_{0}, w_{0}\right) \in \dot{B}_{p, \infty}^{-2+n / p}\left(\mathbb{R}^{n}\right)$ and $\left\|\left(v_{0}, w_{0}\right)\right\|_{\dot{B}_{p, q}^{-2+n / p}} \leq \varepsilon$, where $\varepsilon$ is as above. Suppose furthermore that $v_{0}, w_{0}$ are homogeneous of degree -2 , i.e., they satisfy the relations $v_{0}(x)=\lambda^{2} v_{0}(\lambda x)$ and $w_{0}(x)=\lambda^{2} w_{0}(\lambda x)$ for all $x \in \mathbb{R}^{n}$ and $\lambda>0$. Then the unique global solution ensured by Theorem 1.1 is a self-similar solution, i.e., it satisfies the following condition:

$$
v(x, t)=\lambda^{2} v\left(\lambda x, \lambda^{2} t\right), \quad w(x, t)=\lambda^{2} w\left(\lambda x, \lambda^{2} t\right), \quad \phi(x, t)=\phi\left(\lambda x, \lambda^{2} t\right) .
$$

In the next section we give the proof of Theorem 1.1.

\section{The proof of Theorem 1.1}

We first recall some basic notions and preliminary results used in the proof of Theorem 1.1. Let $\mathcal{S}\left(\mathbb{R}^{n}\right)$ be the Schwartz space and $\mathcal{S}^{\prime}\left(\mathbb{R}^{n}\right)$ be its dual. Given $f \in \mathcal{S}\left(\mathbb{R}^{n}\right)$, the Fourier transform of it, $\mathcal{F}(f)=\widehat{f}$, is defined by

$$
\mathcal{F}(f)(\xi)=\widehat{f}(\xi)=\frac{1}{(2 \pi)^{n / 2}} \int_{\mathbb{R}^{n}} f(x) e^{-i x \cdot \xi} d x
$$

Let $\mathcal{D}_{1}=\left\{\xi \in \mathbb{R}^{n},|\xi| \leq \frac{4}{3}\right\}$ and $\mathcal{D}_{2}=\left\{\xi \in \mathbb{R}^{n}, \frac{3}{4} \leq|\xi| \leq \frac{8}{3}\right\}$. Choose two non-negative functions $\phi, \psi \in \mathcal{S}\left(\mathbb{R}^{n}\right)$ supported, respectively, in $\mathcal{D}_{1}$ and $\mathcal{D}_{2}$ such that

$$
\begin{gathered}
\psi(\xi)+\sum_{j \geq 0} \phi\left(2^{-j} \xi\right)=1, \quad \xi \in \mathbb{R}^{n}, \\
\sum_{j \in \mathbb{Z}} \phi\left(2^{-j} \xi\right)=1, \quad \xi \in \mathbb{R}^{n} \backslash\{0\} .
\end{gathered}
$$


We denote $\phi_{j}(\xi)=\phi\left(2^{-j} \xi\right), h=\mathcal{F}^{-1} \phi$ and $\tilde{h}=\mathcal{F}^{-1} \psi$, where $\mathcal{F}^{-1}$ is the inverse Fourier transform. Then the dyadic blocks $\Delta_{j}$ and $S_{j}$ can be defined as follows:

$$
\begin{aligned}
& \Delta_{j} f=\phi\left(2^{-j} D\right) f=2^{j n} \int_{\mathbb{R}^{n}} h\left(2^{j} y\right) f(x-y) d y, \\
& S_{j} f=\psi\left(2^{-j} D\right) f=2^{j n} \int_{\mathbb{R}^{n}} \tilde{h}\left(2^{j} y\right) f(x-y) d y .
\end{aligned}
$$

Here $D=\left(D_{1}, D_{2}, \cdots, D_{n}\right)$ and $D_{j}=i^{-1} \partial_{x_{j}}\left(i^{2}=-1\right)$. The set $\left\{\Delta_{j}, S_{j}\right\}_{j \in \mathbb{Z}}$ is called the Littlewood-Paley decomposition. Formally, $\Delta_{j}=S_{j}-S_{j-1}$ is a frequency projection to the annulus $\left\{|\xi| \sim 2^{j}\right\}$, and $S_{j}=\sum_{k \leq j-1} \Delta_{k}$ is a frequency projection to the ball $\left\{|\xi| \leq 2^{j}\right\}$. For more details, please reader to [4] and [11]. Let $\mathcal{Z}\left(\mathbb{R}^{n}\right)=$ $\left\{f \in \mathcal{S}\left(\mathbb{R}^{n}\right): \partial^{\alpha} \widehat{f}(0)=0, \forall \alpha \in(\mathbb{N} \cup\{0\})^{n}\right\}$, and denote by $\mathcal{Z}^{\prime}\left(\mathbb{R}^{n}\right)$ the dual of it. Recall that for $s \in \mathbb{R}$ and $(p, q) \in[1, \infty] \times[1, \infty]$, the homogeneous Besov space $\dot{B}_{p, q}^{s}\left(\mathbb{R}^{n}\right)$ is defined by

$$
\dot{B}_{p, q}^{s}\left(\mathbb{R}^{n}\right)=\left\{f \in \mathcal{Z}^{\prime}\left(\mathbb{R}^{n}\right):\|f\|_{\dot{B}_{p, q}^{s}}<\infty\right\}
$$

where

$$
\|f\|_{\dot{B}_{p, q}^{s}}= \begin{cases}\left(\sum_{j \in \mathbb{Z}} 2^{j s q}\left\|\Delta_{j} f\right\|_{L^{p}}^{q}\right)^{1 / q} & \text { for } 1 \leq q<\infty, \\ \sup _{j \in \mathbb{Z}} 2^{j s}\left\|\Delta_{j} f\right\|_{L^{p}} & \text { for } q=\infty .\end{cases}
$$

It is well-known that if either $s<\frac{n}{p}$ or $s=\frac{n}{p}$ and $q=1$, then $\left(\dot{B}_{p, q}^{s}\left(\mathbb{R}^{n}\right), \|\right.$. $\left.\|_{\dot{B}_{p, q}^{s}}\right)$ is a Banach space. Moreover, if we denote $D^{s} f=\mathcal{F}^{-1}\left(|\xi|^{s} \mathcal{F}(f)\right)$, then for any function $f$ defined on $\mathbb{R}^{n} \backslash\{0\}$ which is smooth and homogeneous of degree $k$, the corresponding pseudo-differential operator $f(D)$ is a bounded linear map from $\dot{B}_{p, q}^{s}\left(\mathbb{R}^{n}\right)$ to $\dot{B}_{p, q}^{s-k}\left(\mathbb{R}^{n}\right)$. Besides, there exists a constant $C$ depending only on the dimension $n$ such that for any $s>0, j \in \mathbb{Z}$ and $1 \leq p \leq q \leq \infty$, there holds the following Bernstein inequality:

$$
\operatorname{supp} \widehat{f} \subset\left\{|\xi| \leq 2^{j}\right\} \quad \Longrightarrow\left\|D^{s} f\right\|_{L^{q}} \leq C 2^{j s+j n(1 / p-1 / q)}\|f\|_{L^{p}} .
$$

We now recall the definition of the Chemin-Lerner space $\mathfrak{L}^{r}\left(0, T ; \dot{B}_{p, q}^{s}\left(\mathbb{R}^{n}\right)\right)$ :

Definition 2.1. ([4]) Let $s \in \mathbb{R}, 1 \leq p, q, r \leq \infty$, and $0<T \leq \infty$ be fixed. The Chemin-Lerner space is defined by

$$
\mathfrak{L}^{r}\left(0, T ; \dot{B}_{p, q}^{s}\left(\mathbb{R}^{n}\right)\right):=\left\{f \in \mathcal{D}^{\prime}\left((0, T), \mathcal{Z}^{\prime}\left(\mathbb{R}^{n}\right)\right):\|f\|_{\mathfrak{L}^{r}\left(0, T ; \dot{B}_{p, q}^{s}\left(\mathbb{R}^{n}\right)\right)}<\infty\right\},
$$

where $\|f\|_{\mathfrak{L}^{r}\left(0, T ; \dot{B}_{p, q}^{s}\right)}=\left(\sum_{j \in \mathbb{Z}} 2^{j s q}\left\|\Delta_{j} f\right\|_{L^{r}\left(0, T ; L^{p}\right)}^{q}\right)^{1 / q}$.

We define the usual space $L^{r}\left(0, T ; \dot{B}_{p, q}^{s}\left(\mathbb{R}^{n}\right)\right)$ associated with the norm

$$
\|f\|_{L^{r}\left(0, T ; \dot{B}_{p, q}^{s}\right)}=\left(\int_{0}^{T}\left(\sum_{j \in \mathbb{Z}} 2^{j s q}\left\|\Delta_{j} f\right\|_{L^{p}}^{q}\right)^{r / q} d t\right)^{1 / r} .
$$


By the Minkowski inequality, it is readily to verify that

$$
\begin{cases}\|f\|_{\mathfrak{L}^{r}\left(0, T ; \dot{B}_{p, q}^{s}\right)} \leq\|f\|_{L^{r}\left(0, T ; \dot{B}_{,, q}^{s}\right)} & \text { if } \quad r \leq q, \\ \|f\|_{L^{r}\left(0, T ; \dot{B}_{p, q}^{s}\right)} \leq\|f\|_{\mathfrak{L}^{r}\left(0, T ; \dot{B}_{p, q}^{s}\right)} & \text { if } \quad q \leq r .\end{cases}
$$

In our discussion we shall use two basic results related to this space. The first one is concerned with the product of two functions in this space and reads as follows:

Lemma 2.2. ([5, 14]) Let $1 \leq p, q, r, r_{1}, r_{2} \leq \infty, s_{1}, s_{2}<\frac{n}{p}, s_{1}+s_{2}>0$ and $\frac{1}{r}=$ $\frac{1}{r_{1}}+\frac{1}{r_{2}}$. Then there exists a positive constant $C$ depending only on $s_{1}, s_{2}, p, q, r, r_{1}, r_{2}$ and $n$ such that

$$
\|f g\|_{\mathfrak{L}^{r}\left(0, T ; \dot{B}_{p, q}^{s_{1}+s_{2}-n / p}\right)} \leq C\|f\|_{\mathfrak{L}^{r_{1}}\left(0, T ; \dot{B}_{p, q}^{s_{1}}\right)}\|g\|_{\mathfrak{L}^{r_{2}\left(0, T ; \dot{B}_{p, q}^{s_{2}}\right)}} .
$$

The proof of this result is a simple application of the following fundamental result concerning the product of two functions in the homogeneous Besov spaces: Let $1 \leq p, q \leq \infty, s_{1}, s_{2}<\frac{n}{p}$ and $s_{1}+s_{2}>0$. Then there exists a constant $C$ depending only on $p, q, s_{1}, s_{2}$ and $n$ such that

$$
\|f g\|_{\dot{B}_{p, q}^{s_{1}+s_{2}-n / p}} \leq C\|f\|_{\dot{B}_{p, q}^{s_{1}}}\|g\|_{\dot{B}_{p, q}^{s_{2}}} .
$$

For details of the proof, we refer the reader to see [5] and [14]. The second one, whose proof can be found from e.g. [5], is concerned with the Cauchy problem of the heat equation:

$$
\left\{\begin{array}{l}
\frac{\partial u}{\partial t}-\Delta u=f(x, t), \quad x \in \mathbb{R}^{n}, t>0, \\
u(x, 0)=u_{0}(x), \quad x \in \mathbb{R}^{n} .
\end{array}\right.
$$

Proposition 2.3. ([5]) Let $s \in \mathbb{R}, 1 \leq p, q, r_{1} \leq \infty$ and $0<T \leq \infty$. Assume that $u_{0} \in \dot{B}_{p, q}^{s}\left(\mathbb{R}^{n}\right)$ and $f \in \mathfrak{L}^{r_{1}}\left(0, T ; \dot{B}_{p, q}^{s+2 / r_{1}-2}\left(\mathbb{R}^{n}\right)\right)$. Then (2.3) has a unique solution $u \in \underset{r_{1} \leq r \leq \infty}{\cap} \mathfrak{L}^{r}\left(0, T ; \dot{B}_{p, q}^{s+2 / r}\left(\mathbb{R}^{n}\right)\right)$. In addition, there exists a constant $C>0$ depending only on $n$ such that for any $r_{1} \leq r \leq \infty$, we have

$$
\|u\|_{\mathfrak{L}^{r}\left(0, T ; \dot{B}_{p, q}^{s+2 / r}\right)} \leq C\left(\left\|u_{0}\right\|_{\dot{B}_{p, q}^{s}}+\|f\|_{\mathfrak{L}^{r_{1}}\left(0, T ; \dot{B}_{p, q}^{s+2 / r_{1}-2}\right)}\right) .
$$

Next we recall an existence and uniqueness result for an abstract operator equation in a generic Banach space. For the proof we refer the reader to see LemariéRieusset [11].

Proposition 2.4. ([1]) Let $\mathcal{X}$ be a Banach space and $\mathbf{B}: \mathcal{X} \times \mathcal{X} \rightarrow \mathcal{X}$ is a bilinear bounded operator, $\|\cdot\|_{\mathcal{X}}$ being the $\mathcal{X}$-norm. Assume that for any $u_{1}, u_{2} \in \mathcal{X}$, we have $\left\|\mathbf{B}\left(u_{1}, u_{2}\right)\right\|_{\mathcal{X}} \leq C_{0}\left\|u_{1}\right\|_{\mathcal{X}}\left\|u_{2}\right\|_{\mathcal{X}}$. Then for any $y \in \mathcal{X}$ such that $\|y\|_{\mathcal{X}} \leq \varepsilon<\frac{1}{4 C_{0}}$, the equation $u=y+\mathbf{B}(u, u)$ has a solution $u$ in $\mathcal{X}$. Moreover, this solution is the only one such that $\|u\|_{\mathcal{X}} \leq 2 \varepsilon$, and depends continuously on $y$ in the following sense: if $\|\widetilde{y}\|_{\mathcal{X}} \leq \varepsilon, \widetilde{u}=\widetilde{y}+\mathbf{B}(\widetilde{u}, \widetilde{u})$ and $\|\widetilde{u}\|_{\mathcal{X}} \leq 2 \varepsilon$, then $\|u-\widetilde{u}\|_{\mathcal{X}} \leq \frac{1}{1-4 \varepsilon C_{0}}\|y-\widetilde{y}\|_{\mathcal{X}}$. 
We are now ready to give the proof of Theorem 1.1. Let $p$ and $q$ be as in Theorem 1.1, i.e., $2 \leq p<2 n$ and $1 \leq q \leq \infty$, and let $r$ be as in (1.4), i.e., $1<r \leq \infty$. We choose a number $2<r_{1} \leq 2 r$ such that $\frac{2}{r_{1}}+\frac{n}{p}>\frac{3}{2}$. For $T>0$ to be specified later, we set $\mathcal{X}_{T}=\mathfrak{L}^{r_{1}}\left(0, T ; \dot{B}_{p, q}^{-2+n / p+2 / r_{1}}\left(\mathbb{R}^{n}\right)\right)$. Given $(v, w) \in \mathcal{X}_{T}$, we define $\mathcal{G}(v, w)=(\bar{v}, \bar{w})$ to be the solution of the following initial value problem:

$$
\begin{array}{ll}
\partial_{t} \bar{v}-\Delta \bar{v}=-\nabla \cdot\left(v \nabla(-\Delta)^{-1}(w-v)\right), & \bar{v}(x, 0)=v_{0}(x), \\
\partial_{t} \bar{w}-\Delta \bar{w}=\nabla \cdot\left(w \nabla(-\Delta)^{-1}(w-v)\right), & \bar{w}(x, 0)=w_{0}(x) .
\end{array}
$$

Obviously, $(v, w)$ is a solution of (1.3) if and only if it is a fixed point of $\mathcal{G}$.

Lemma 2.5. Let $(v, w) \in \mathcal{X}_{T}$. Then $(\bar{v}, \bar{w}) \in \mathcal{X}_{T}$. Moreover, there exists a constant $C_{0}>0$ such that

$$
\begin{aligned}
& \|\bar{v}\|_{\mathcal{X}_{T}} \leq\left\|e^{t \Delta} v_{0}\right\|_{\mathcal{X}_{T}}+C_{0}\|(v, w)\|_{\mathcal{X}_{T}}^{2}, \\
& \|\bar{w}\|_{\mathcal{X}_{T}} \leq\left\|e^{t \Delta} w_{0}\right\|_{\mathcal{X}_{T}}+C_{0}\|(v, w)\|_{\mathcal{X}_{T}}^{2}
\end{aligned}
$$

Here $e^{t \Delta}$ is the heat operator with kernel $G(x, t)=(4 \pi t)^{-n / 2} \exp \left(-\frac{|x|^{2}}{4 t}\right)$.

Proof. By Duhamel principle, (2.5) is equivalent to the following integral equation:

$$
\bar{v}(t)=e^{t \Delta} v_{0}-\int_{0}^{t} e^{(t-\tau) \Delta} \nabla \cdot\left(v \nabla(-\Delta)^{-1}(w-v)\right)(\tau) d \tau
$$

Since $2 \leq p<2 n, 2<r_{1}<\infty$ and $\frac{n}{p}+\frac{2}{r_{1}}>\frac{3}{2}$, by choosing $s_{1}=-2+\frac{n}{p}+\frac{2}{r_{1}}$ and $s_{2}=-1+\frac{n}{p}+\frac{2}{r_{1}}$ in Lemma 2.2 we get

$$
\begin{aligned}
\| \nabla \cdot(v & \left.\nabla(-\Delta)^{-1}(w-v)\right) \|_{\mathfrak{L}^{r_{1} / 2}\left(0, T ; \dot{B}_{p, q}^{-4+n / p+4 / r_{1}}\right)} \\
& \leq C\left\|v \nabla(-\Delta)^{-1}(w-v)\right\|_{\mathfrak{L}^{r_{1} / 2}\left(0, T ; \dot{B}_{p, q}^{-3+n / p+4 / r_{1}}\right)} \\
& \leq C\|v\|_{\mathfrak{L}^{r_{1}}\left(0, T ; \dot{B}_{p, q}^{-2+n / p+2 / r_{1}}\right)}\left\|\nabla(-\Delta)^{-1}(w-v)\right\|_{\mathfrak{L}^{r_{1}}\left(0, T ; \dot{B}_{p, q}^{-1+n / p+2 / r_{1}}\right)} \\
& \leq C\|(v, w)\|_{\mathfrak{L}^{r_{1}}\left(0, T ; \dot{B}_{p, q}^{-2+n / p+2 / r_{1}}\right)}^{2}
\end{aligned}
$$

Hence, by using Proposition 2.3 we conclude that there exists a positive constant $C_{0}$ such that

$$
\begin{aligned}
\|\bar{v}\|_{\mathcal{X}_{T}} & \leq\left\|e^{t \Delta} v_{0}\right\|_{\mathcal{X}_{T}}+C\left\|\nabla \cdot\left(v \nabla(-\Delta)^{-1}(w-v)\right)\right\|_{\mathfrak{L}^{r_{1} / 2}\left(0, T ; \dot{B}_{p, q}^{-4+n / p+4 / r_{1}}\right)} \\
& \leq\left\|e^{t \Delta} v_{0}\right\|_{\mathcal{X}_{T}}+C_{0}\|(v, w)\|_{\mathfrak{L}^{r_{1}}\left(0, T ; \dot{B}_{p, q}^{-2+n / p+2 / r_{1}}\right)}^{2} \cdot
\end{aligned}
$$

This proves (2.7). The proof of (2.8) is similar. 
Proof of Theorem 1.1: The above lemma ensures that $\mathcal{G}$ is well-defined and maps $\mathcal{X}_{T}$ into itself. Moreover, from (2.7) and (2.8) we see that for any $(v, w) \in \mathcal{X}_{T}$ and $(\bar{v}, \bar{w})=\mathcal{G}(v, w)$,

$$
\|(\bar{v}, \bar{w})\|_{\mathcal{X}_{T}} \leq\left\|\left(e^{t \Delta} v_{0}, e^{t \Delta} w_{0}\right)\right\|_{\mathcal{X}_{T}}+C_{0}\|(v, w)\|_{\mathcal{X}_{T}}^{2} .
$$

Existence. We first prove global existence for small initial data. For this purpose we choose $T=\infty$. By Proposition 2.4 and (2.11) it is easy to see that if $\left\|\left(e^{t \Delta} v_{0}, e^{t \Delta} w_{0}\right)\right\|_{\mathcal{X}_{\infty}} \leq \varepsilon$ and $\varepsilon>0$ is so small that $4 C_{0} \varepsilon \leq 1$, then $\mathcal{G}$ has a fixed point in the closed ball $\|(v, w)\|_{\mathcal{X}_{\infty}} \leq 2 \varepsilon$ in $\mathcal{X}_{\infty}$. By Proposition 2.3 we see that the condition $\left\|\left(e^{t \Delta} v_{0}, e^{t \Delta} w_{0}\right)\right\|_{\mathcal{X}_{\infty}} \leq \varepsilon$ is satisfied if $\left\|\left(v_{0}, w_{0}\right)\right\|_{\dot{B}_{p, q}^{-2+n / p}}$ is small enough. Indeed, by Proposition 2.3, there exists a positive constant $C_{1}$ depending only on $n$ such that $\left\|\left(e^{t \Delta} v_{0}, e^{t \Delta} w_{0}\right)\right\|_{\mathcal{X}_{\infty}} \leq C_{1}\left\|\left(v_{0}, w_{0}\right)\right\|_{\dot{B}_{p, q}^{-2+n / p}}$. Hence, if we assume that $\left\|\left(v_{0}, w_{0}\right)\right\|_{\dot{B}_{p, q}^{-2+n / p}} \leq C_{1}^{-1} \varepsilon$, then we have $\left\|\left(e^{t \Delta} v_{0}, e^{t \Delta} w_{0}\right)\right\|_{\mathcal{X}_{\infty}} \leq \varepsilon$. This proves global existence for small initial data.

Next we prove local existence for large initial data. For this purpose we split $v_{0}$ into a sum as follows: $\widehat{v}_{0}(\xi)=\widehat{v}_{0} 1_{\left\{|\xi|>2^{N}\right\}}+\widehat{v}_{0} 1_{\left\{|\xi| \leq 2^{N}\right\}}:=\widehat{v_{01}}+\widehat{v_{02}}$, where $1_{\mathcal{D}}$ represents the characteristic function on the domain $\mathcal{D}$. Similarly, we split $w_{0}$ as $\widehat{w_{0}}=\widehat{w_{01}}+\widehat{w_{02}}$. Since $2 \leq p<2 n$, it is easy to see that if $N \in \mathbb{Z}^{+}$is sufficiently large then $C_{1}\left\|\left(v_{01}, w_{01}\right)\right\|_{\dot{B}_{p, q}^{-2+n / p}} \leq \frac{1}{2} \varepsilon$. Choosing a such $N$ and fixing it, we get

$$
\left\|\left(e^{t \Delta} v_{0}, e^{t \Delta} w_{0}\right)\right\|_{\mathcal{X}_{T}} \leq \frac{1}{2} \varepsilon+\left\|\left(e^{t \Delta} v_{02}, e^{t \Delta} w_{02}\right)\right\|_{\mathcal{X}_{T}}
$$

Applying the Bernstein inequality,

$$
\begin{aligned}
& \left\|\left(e^{t \Delta} v_{02}, e^{t \Delta} w_{02}\right)\right\|_{\mathcal{X}_{T}}=\left\|\left(e^{t \Delta} v_{02}, e^{t \Delta} w_{02}\right)\right\|_{\mathfrak{L}^{r_{1}}\left(0, T ; \dot{B}_{p, q}^{-2+n / p+2 / r_{1}}\right)} \\
& \quad \lesssim 2^{(2 N) / r_{1}}\left\|\left(e^{t \Delta} v_{02}, e^{t \Delta} w_{02}\right)\right\|_{\mathfrak{L}^{r_{1}}\left(0, T ; \dot{B}_{p, q}^{-2+n / p}\right)} \leq C_{2} 2^{(2 N) / r_{1}} T^{1 / r_{1}}\left\|\left(v_{0}, w_{0}\right)\right\|_{\dot{B}_{p, q}^{-2+n / p}} .
\end{aligned}
$$

Hence, if we choose $T$ small enough such that $C_{2} 2^{(2 N) / r_{1}} T^{1 / r_{1}}\left\|\left(v_{0}, w_{0}\right)\right\|_{\dot{B}_{p, q}^{-2+n / p}} \leq$ $\frac{1}{2} \varepsilon$, then $\left\|\left(e^{t \Delta} v_{02}, e^{t \Delta} w_{02}\right)\right\|_{\mathcal{X}_{T}} \leq \frac{\varepsilon}{2}$. This result together with (2.12) yields that $\left\|\left(e^{t \Delta} v_{0}, e^{t \Delta} w_{0}\right)\right\|_{\mathcal{X}_{T}} \leq \varepsilon$. By applying Proposition 2.4 again, we obtain a fixed point of $\mathcal{G}$ in the closed ball $\|(v, w)\|_{\mathcal{X}_{T}} \leq 2 \varepsilon$ in $\mathcal{X}_{T}$, and concludes the proof of local existence of solution.

Regularity. Note that if $(v, w) \in \mathcal{X}_{T}$ is a solution of (1.1), then we can proceed in the same way as in the proof of Lemma 2.5 to obtain that

$$
\nabla \cdot\left(v \nabla(-\Delta)^{-1}(w-v)\right), \nabla \cdot\left(w \nabla(-\Delta)^{-1}(w-v)\right) \in \mathfrak{L}^{r_{1} / 2}\left(0, T ; \dot{B}_{p, q}^{-4+n / p+4 / r_{1}}\left(\mathbb{R}^{n}\right)\right) .
$$

By Proposition 2.3, for any $\frac{r_{1}}{2} \leq r \leq \infty$ we have $(v, w) \in \mathfrak{L}^{r}\left(0, T ; \dot{B}_{p, q}^{-2+n / p+2 / r}\left(\mathbb{R}^{n}\right)\right)$. Moreover, if $\left(v_{0}, w_{0}\right)$ belongs to the closure of $\mathcal{S}\left(\mathbb{R}^{n}\right)$ in the space $\dot{B}_{p, q}^{-2+n / p}\left(\mathbb{R}^{n}\right)$, then $(v, w) \in C\left([0, T), \dot{B}_{p, q}^{-2+n / p}\left(\mathbb{R}^{n}\right)\right)$. 
Uniqueness. Let $(v, w)$ and $(\tilde{v}, \tilde{w})$ be two solutions of (1.1) in $\mathcal{X}_{T}$ associated with the initial data $\left(v_{0}, w_{0}\right)$ and $\left(\tilde{v}_{0}, \tilde{w}_{0}\right)$, respectively. Set $V=v-\tilde{v}, W=w-\tilde{w}$. Then $(V, W)$ satisfies the following equations:

$$
\left\{\begin{array}{l}
\partial_{t} V-\Delta V=-\nabla \cdot\left(V \nabla(-\Delta)^{-1}(w-v)\right)-\nabla \cdot\left(\tilde{v} \nabla(-\Delta)^{-1}(W-V)\right), \\
\partial_{t} W-\Delta W=\nabla \cdot\left(W \nabla(-\Delta)^{-1}(w-v)\right)+\nabla \cdot\left(\tilde{w} \nabla(-\Delta)^{-1}(W-V)\right), \\
V(x, 0)=V_{0}(x)=v_{0}(x)-\tilde{v}_{0}(x), \quad W(x, 0)=W_{0}(x)=w_{0}(x)-\tilde{w}_{0}(x) .
\end{array}\right.
$$

Proceeding in the same way as in the proof of Lemma 2.5, we can prove that

$$
\begin{aligned}
& \left\|-\nabla \cdot\left(V \nabla(-\Delta)^{-1}(w-v)\right)-\nabla \cdot\left(\tilde{v} \nabla(-\Delta)^{-1}(W-V)\right)\right\|_{\mathcal{X}_{T}} \\
& \quad \leq C\left(\|v\|_{\mathcal{X}_{T}}+\|\tilde{v}\|_{\mathcal{X}_{T}}+\|w\|_{\mathcal{X}_{T}}\right)\|(V, W)\|_{\mathcal{X}_{T}} \\
& \left\|\nabla \cdot\left(W \nabla(-\Delta)^{-1}(w-v)\right)+\nabla \cdot\left(\tilde{w} \nabla(-\Delta)^{-1}(W-V)\right)\right\|_{\mathcal{X}_{T}} \\
& \quad \leq C\left(\|v\|_{\mathcal{X}_{T}}+\|w\|_{\mathcal{X}_{T}}+\|\tilde{w}\|_{\mathcal{X}_{T}}\right)\|(V, W)\|_{\mathcal{X}_{T}} .
\end{aligned}
$$

Hence, by Proposition 2.3,

$$
\begin{aligned}
\|(V, W)\|_{\mathcal{X}_{T}} & \leq C_{1}\left\|\left(V_{0}, W_{0}\right)\right\|_{\dot{B}_{p, q}^{-2+n / p}} \\
& +C_{0}\left(\|v\|_{\mathcal{X}_{T}}+\|\tilde{v}\|_{\mathcal{X}_{T}}+\|w\|_{\mathcal{X}_{T}}+\|\tilde{w}\|_{\mathcal{X}_{T}}\right)\|(V, W)\|_{\mathcal{X}_{T}} .
\end{aligned}
$$

Let $M(T):=C_{0}\left(\|v\|_{\mathcal{X}_{T}}+\|\tilde{v}\|_{\mathcal{X}_{T}}+\|w\|_{\mathcal{X}_{T}}+\|\tilde{w}\|_{\mathcal{X}_{T}}\right)$. By absolute continuity of the Lebesgue integral, we have that $M(T)$ converges to zero as $T \rightarrow 0^{+}$. Hence, if we choose $T_{1}$ sufficiently small such that $M\left(T_{1}\right) \leq \frac{1}{2}$, then

$$
\|(V, W)\|_{\mathcal{X}_{T}} \leq 2 C_{1}\left\|\left(V_{0}, W_{0}\right)\right\|_{\dot{B}_{p, q}^{-2+n / p}}
$$

Repeating this argument step by step on the intervals $\left[0, T_{1}\right),\left[T_{1}, 2 T_{1}\right), \ldots$, we finally get a constant $C=C_{T}$ after a finite steps such that $\|(V, W)\| \mathcal{X}_{T} \leq C\left\|\left(V_{0}, W_{0}\right)\right\|_{\dot{B}_{p, q}^{-2+n / p} \text {. }}$ This proves (1.5) which implies the uniqueness of solutions. The proof of Theorem 1.1 is complete.

Acknowledgment. This work is supported by the China National Natural Science Fundation under the Grant No.10771223.

\section{References}

[1] N. Ben Abdallah, F. Méhats, N. Vauchelet, A note on the long time behavior for the drift-diffusion-Poisson system, C. R. Math. Acad. Sci. Paris, 339 (10) (2004) 683-688.

[2] P. Biler, J. Dolbeault, Long time behavior of solutions to Nernst-Planck and DebyeHückel drift-diffusion systems, Ann. Henri Poincaré, 1 (2000) 461-472.

[3] P. Biler, W. Hebisch, T. Nadzieja, The Debye system: existence and large time behavior of solutions, Nonlinear Anal., 23 (1994) 1189-1209. 
[4] J.-Y. Chemin, Perfect Incompressible Fluids, Oxford Lecture Series in Mathematics and its Applications, vol. 14. The Clarendon Press, Oxford University Press: New York, 1998.

[5] R. Dachin, Fourier Analysis Methods for PDE's, 2005, http://perso-math.univ-mlv.fr/users/danchin.raphael/courschine.pdf.

[6] P. Debye, E. Hückel, Zur Theorie der Elektrolyte, II: Das Grenzgesetz für die elektrische Leitfähigkeit, Phys. Z., 24 (1923) 305-325.

[7] H. Gajewski, On existence, uniqueness and asymptotic behavior of solutions of the basic equations for carrier transport in semiconductors, Z. Angew. Math. Mech., 65 (1985) 101-108.

[8] H. Gajewski, K. Gröger, On the basic equations for carrier transport in semiconductors, J. Math. Anal. Appl., 113 (1986) 12-35.

[9] G. Karch, Scaling in nonlinear parabolic equations, J. Math. Anal. Appl., 234 (1999) $534-558$.

[10] M. Kurokiba, T. Ogawa, Well-posedness for the drift-diffusion system in $L^{p}$ arising from the semiconductor device simulation, J. Math. Anal. Appl., 342 (2008) 1052-1067.

[11] P.-G. Lemarié-Rieusset, Recent Developments in the Navier-Stokes Problem, Research Notes in Mathematics, Chapman \& Hall/CRC, 2002.

[12] M. S. Mock, An initial value problem from semiconductor device theory, SIAM J. Math. Anal., 5 (1974) 597-612.

[13] T. Ogawa, S. Shimizu, The drift-diffusion system in two-dimensional critical Hardy space, J. Funct. Anal., 255 (2008) 1107-1138.

[14] T. Runst, W. Sickel, Sobolev Spaces of Fractional Order, Nemytskij Operators, and Nonlinear Partial Differential Equations, de Gruyter Series in Nonlinear Analysis and Applications, vol. 3. Walter de Gruyter \& Co.: Berlin, 1996.

[15] S. Selberherr, Analysis and simulation of semiconductor devices, Springer Verlag, 1983. 